\title{
A note on the use of ellipsometry for studying the kinetics of formation of self-assembled monolayers
}

\author{
MURALI SASTRY \\ Materials Chemistry Division, National Chemical Laboratory, Pune 411 008, India
}

MS received 22 December 1999; revised 6 April 2000

\begin{abstract}
Ellipsometry is currently one of the most important techniques for characterization of the deposition and growth mode of ultra thin organic films. However, it is well known that for thicknesses normally encountered in organic monolayer films, as would occur for example in self-assembled monolayers, ellipsometry cannot be used to simultaneously determine the thickness and refractive index of the monolayer film. Current practice is to assume a reasonable value for the film refractive index and calculate an effective 'ellipsometric thickness'. This communication seeks to show that the alternative approach of assuming a thickness for the monolayer (determined by the length of the molecule) and calculating the effective film refractive index lends itself to easier and more meaningful physical interpretation. The Lorentz-Lorenz formula is then used to transform the effective refractive index into a surface coverage and hence to an effective mass coverage. The methodology advanced is applied to the kinetics of formation of a self-assembled monolayer of a well-studied molecule, octadecanethiol on Au.
\end{abstract}

Keywords. Ellipsometry; self-assembled monolayers; organic thin films; Lorentz-Lorenz formula.

\section{Introduction}

A very important aspect of the study of self-assembled monolayers (SAMs), an area with much technological promise (Kaeble 1971; Notoya and Poling 1979; Durand et al 1983; Lopez et al 1993), is the determination of correct protocol for film formation. Protocol implies laying down ideal conditions for formation of a compact, close-packed molecular assembly and is determined by parameters such as monomer concentration in the solvent, solvent temperature, nature of the substrate on which SAM formation is sought, time of immersion of substrate into the solvent etc. With newer and newer molecules found to be capable of self-assembly (Sun et al 1990; Colvin et al 1992), this aspect of research assumes greater importance.

Ellipsometry, a technique based on the principle of change in state of polarization of light on reflection from an optically flat surface (Azzam and Bashara 1977), is one of the most versatile and commonly used techniques for studying various processes such as the kinetics of SAM formation on metal surfaces (Tillman et al 1988; Bain et al 1989; Wasserman et al 1989), formation of multilayer Langmuir Blodgett films (Honig and De Koning 1976) and protein absorption in biological films (Cuypers 1983) to name a few. At very low film thickness, as would occur in the sub-monolayer and monolayer stages of SAM formation, the change in the ellipsometric angle $\psi$ is well below the experimental uncertainty in angle measurement. A simulation based on an air-organic film-substrate three-layer model of the variation in $\psi$ with refractive index (film thickness assumed to be $25 \AA$ and film thickness (refractive index assumed to be 1.45) is shown in figure 1a for a typical SAM forming molecule, octadecanethiol (ODT) on Au. The experimental uncertainty of $0.02^{\circ}$ for the ellipsometer of this study is shown as a bar in the figure and clearly shows that a variation in $\psi$ cannot be measured at such a low film thickness. This effectively rules out the simultaneous determination of two parameters (for example, the film thickness, $t$ and film refractive index, $n$ ) from a single ellipsometry measurement. Recognizing this problem, the current approach adopted by researchers is to assume a reasonable value of the film refractive index and then calculate an effective ellipsometric thickness from a measurement of the ellipsometric angles $\psi$ and $\Delta$. The kinetics of SAM formation would then be represented by an ellipsometric thickness versus time curve as a function of monomer concentration, temperature etc (Tillman et al 1988; Bain et al 1989; Wasserman et al 1989). We suspect that the route adopted may be due to the fact that the variation in $\Delta$ with thickness at constant refractive index is linear (Kim et al 1990) whereas the dependence of $\Delta$ on the refractive index at constant thickness is non-linear, the former approach naturally leading to a certain amount of ease in interpretation and calculation. The above is illustrated in figure 1b which shows a similar three-layer simulation of the deposition on a self-assembled monolayer of octadecanethiol on Au. The above mentioned figure shows the variation of $\Delta$ with thickness (refractive index $=1.45$ ) and 
refractive index (thickness $=25 \AA$, the values of thickness and refractive index for this system being taken from Porter et al (1987). The non-linear dependence of $\Delta$ on the refractive index mentioned above is clearly seen in the figure.

The object of this paper is to examine whether an alternative approach based on determination of the film refractive index assuming a reasonable film thickness would lead to any advantage in interpretation of the kinetics of SAM formation. The non-linearity of $\Delta$ on $n$ is a minor problem given the ease with which ellipsometric calcu-
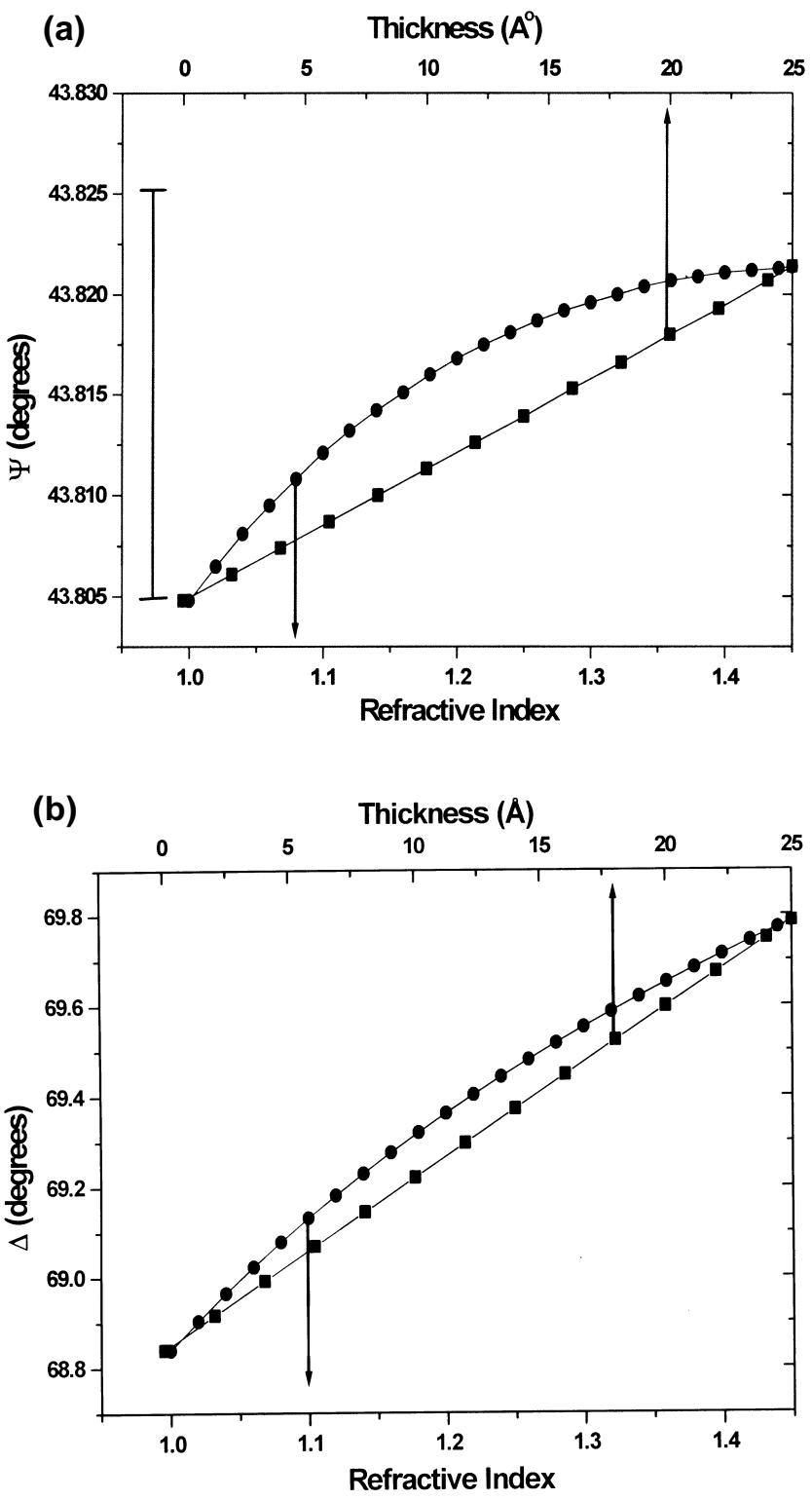

Figure 1. a. Simulation of the variation in $\psi$ with refractive index (circles) and thickness (squares) for an ODT SAM on Au. The corresponding axes are indicated by arrows and $\mathbf{b}$. simulation of the variation in $\Delta$ with refractive index (circles) and thickness (squares) for an ODT SAM on Au. The corresponding axes are indicated by arrows. The experimental uncertainty is shown as a bar in the diagram. lations can be performed on a PC and thus is not an obstacle at all. (All simulations and calculations performed in this paper have been done using Mathcad, a commercial mathematical package for the PC available from Mathsoft Inc., USA). An application has been developed by the author using Mathcad for ellipsometry calculations and is available from the Mathsoft public domain on the Internet). It is well known that the refractive index of a material is a function of its density, leading to the famous Lorentz-Lorenz formula (Born and Wolf 1965; Batsanov 1966; Cuypers et al 1983). We show below that the Lorentz-Lorenz formula can be used to transform the calculated refractive index to a mass coverage (or surface coverage) which gives a much clearer picture of the nature of packing of the molecules in SAM during growth, an interpretation which would not be possible if one determines an effective thickness. This approach also means that adsorption isotherms can be determined from ellipsometry and thermodynamic parameters such as the energy of condensation etc (Chen and Frank 1989) can be determined. Another advantage of this approach is that the mass coverage data thus calculated can be correlated with another independent technique such as quartz crystal microgravimetry (QCM) which has also proved very successful in the study of SAM formation (Hahner et al 1993; Frubose and Doblhofer 1995). We illustrate the methodology by application to the kinetics of SAM formation of octadecanethiol molecules on Au studied by ellipsometry and QCM. We would like to point out here that the main emphasis of the communication is to show the advantages of film refractive index determination from ellipsometry and not to arrive at the protocol for SAM formation with ODT molecules. This is a well-characterized system and we will use conditions detailed in literature (Porter et al 1987).

\section{Experimental}

SAMs of the ODT molecule were formed on thermally evaporated gold-coated glass slides by immersion of the substrate in a $1 \mathrm{mM}$ concentrated solution of the monomer in acetonitrile at room temperature. After thorough washing and drying of the film, ex situ ellipsometry measurements were made on the film surface for different times of immersion to obtain the kinetics of SAM formation. Ellipsometry measurements were performed using a manually operated Gaertner L 119 null ellipsometer operated in the polarizer-compensator-sample-analyzer (PCSA) mode at an angle of incidence of $60^{\circ}$. The compensator was a quarter wave plate set with the optical axis at $45^{\circ}$ to the plane of incidence. The light source was a $\mathrm{He}-\mathrm{Ne}$ laser $(5 \mathrm{~mW})$, the wavelength being $6328 \AA$ ÅMeasurements were made in four zones to correct for any instrument misalignment. A photomultiplier was used to determine the extinction (null) condition precisely. 
The kinetics of SAM formation was also followed simultaneously on a gold-coated AT cut quartz crystal by the standard QCM technique. QCM measurements were performed using an Edwards FTM5 microbalance with a frequency resolution and stability of $\pm 1 \mathrm{~Hz}$. All measurements were performed ex situ for different times of immersion of the quartz crystal after thorough washing and drying of the crystal. QCM measurements are based on the principle that the resonance frequency of an AT-cut quartz crystal changes with mass loading of the crystal surface. The well known Sauerbrey (1959) formula is then used to calculate the mass loading from the change in the resonance frequency during SAM formation. A $6 \mathrm{MHz}$ crystal was used in this study with a mass resolution of $12 \mathrm{ng} / \mathrm{cm}^{2}$.

\section{Results and discussion}

The Lorentz-Lorenz formula (Born and Wolf 1965; Cuypers et al 1983) relates the effective refractive index $n$ of a mixture of substances in the following manner:

$$
\left(n^{2}-1\right) /\left(n^{2}+2\right)=\sum_{i} N_{i} R_{i},
$$

where $N_{i}$ and $R_{i}$ refer to the number of moles/volume and molar refractivity of component $i$ respectively. For a pure substance, the above equation may be transformed into a mass uptake function and may be written as (Cuypers et al 1983):

$$
m=d \rho^{0}=(0 \cdot 1 M d / R)\left[\left(n^{2}-1\right) /\left(n^{2}+2\right)\right],
$$

where $d$ is the thickness of the film in nanometres, $\rho^{0}$ the density of the substance, $M$ the molecular weight of the substance and other parameters are as defined earlier. Thus, using the refractive index determined from ellipsometry (assuming a reasonable film thickness) and (2), one can calculate the mass change on formation of a SAM. This approach is, of course, completely general and may be used to study other ultra thin organic films such as Langmuir Blodgett films (Den Engelsen 1971; Kim et al 1990).

The above ellipsometry analysis has been applied to the kinetics of SAM formation of ODT on Au. Figure 2 shows the kinetics of SAM formation as determined from QCM measurements. The solid curve is an aid to the eye while the error bar corresponds to the instrumental resolution of $\pm 12 \mathrm{ng} / \mathrm{cm}^{2}$. An important parameter that must be included in the QCM mass loading analysis is the quartz crystal surface roughness which would yield a true surface area larger than the measured geometrical area. From cyclic voltammetry measurements, we have determined a surface roughness of $\sim 2 \cdot 2$ for the crystal used in this study which compares favourably with values reported in literature (Grabar et al 1996). We would like to emphasize that an independent determination of crystal surface roughness is extremely important for not only establishing a correlation between QCM and ellipsometry measurements, as investigated here, but also in determining correct protocol for SAM formation using different molecule/substrate systems. The final steady state mass loading of $\sim 205 \mathrm{ng} / \mathrm{cm}^{2}$ taking into account the above roughness factor corresponds to an average molecular area of $23 \AA$ This is slightly larger than the area expected from close packing considerations ( $20 \AA \AA)$. Consequently, the Lorentz-Lorenz relationship for this degree of packing $(R=93.4$ for ODT, molar refractivities for atoms/groups in the molecule were taken from Vogel et al (1954)) gives a refractive index of 1.46 which is close to the value of 1.45 reported in literature (Porter et al 1987). The larger area/molecule observed using QCM may be dictated by the crystallography of the surface, which is an important aspect in SAMs and close-packing considerations may not always apply. The general form of the mass uptake with time curve indicates an initial rapid chemisorption of the ODT molecule followed by slower adsorption which stabilizes within nearly $2 \mathrm{~h}$ of immersion.

The kinetics of SAM formation for the above system was studied ex situ by ellipsometry and the $\Delta$ values were transformed to mass uptake/area using (1) and (2). The film thickness was taken to be 25 Afor calculation of the refractive index. Figure 3 gives the mass loading with time determined ellipsometrically. The error bar corresponds to the experimental uncertainty in measurement of the ellipsometric angles and is close to $\pm 12 \mathrm{ng}$. As mentioned above, the molar refractivity of this molecule is calculated to be 93.4 . It was found that the final steady state conditions gave a refractive index of 1.40 , which leads to a mass loading of $190 \mathrm{ng} / \mathrm{cm}^{2}$ and an average area/molecule of $25 \AA$ This value is slightly larger than that calculated from QCM studies $(23 \AA)$ and larger than

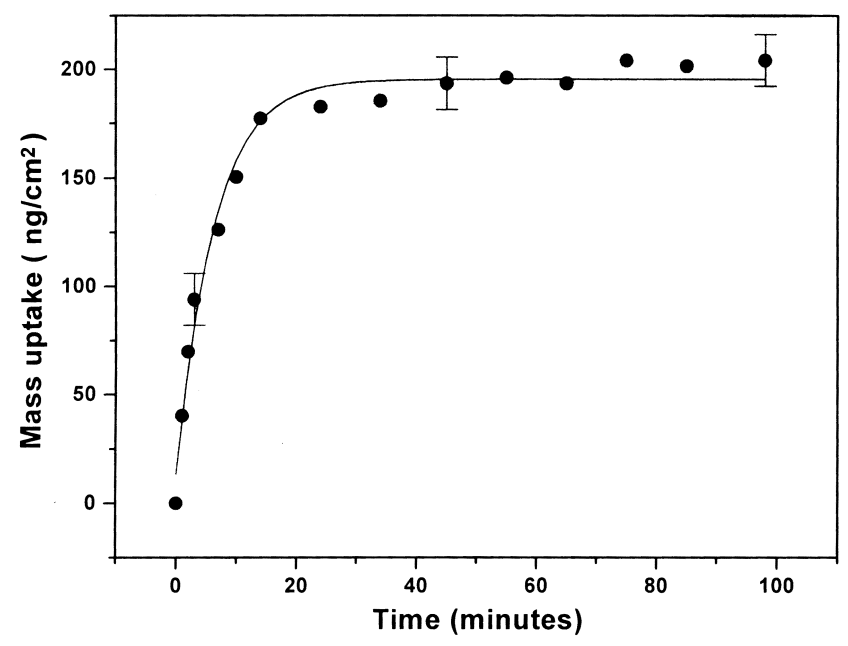

Figure 2. Kinetics of the mass uptake for the ODT SAM on $\mathrm{Au}$ as studied using QCM. A sigmoidal growth curve has been shown to aid the eye. 


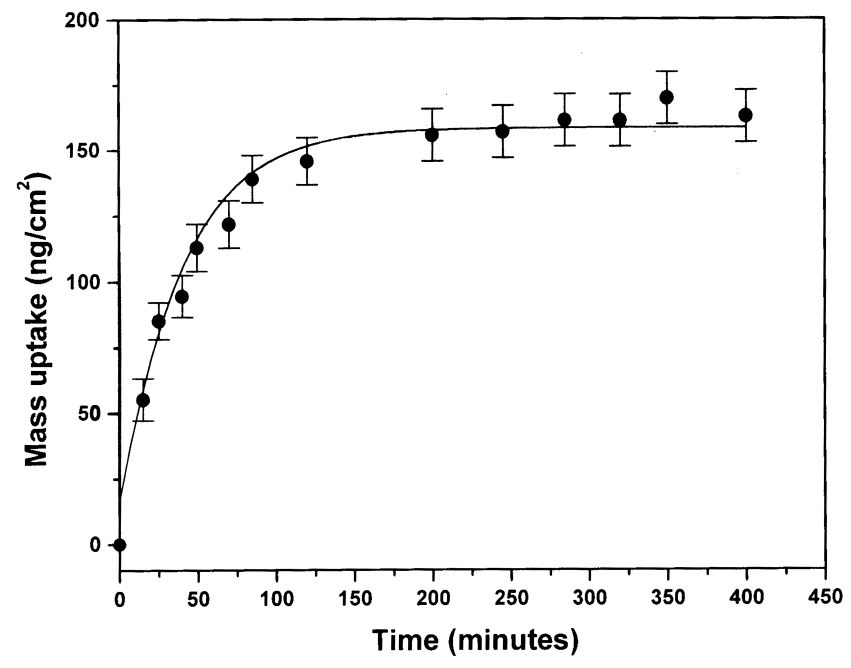

Figure 3. Kinetics of the mass uptake for the ODT SAM as determined from ellipsometry and (1) and (2). A sigmoidal growth curve has been shown to aid the eye.

the area calculated using the Lorentz-Lorenz formula and refractive index $=1.45$ (Porter et al 1987) of $23 \AA$ Comparison of the ODT SAM formation kinetics curves obtained by QCM (figure 2) and ellipsometry (figure 3) reveals the following differences. The equilibrium mass loading in both cases is different as well as the nature of mass uptake with time. These discrepancies may be primarily due to the fact that the dependence of $\psi$ and $\Delta$ on the film refractive index is non-linear. It has been found that the surface crystallography of the gold grains deposited on the AT cut quartz crystal and on glass substrates is quite different and may also be responsible for the difference in kinetics (and final state molecular packing) as observed by the two techniques. While this is in itself an interesting finding and is to be dealt with elsewhere, the main emphasis of this communication is on demonstrating that the alternative route of refractive index determination from ellipsometry has certain inherent benefits as has already been shown through the now possible correlation with QCM measurements.

This approach is more appealing due to its closer correlation with the actual physical situation prevailing during SAM formation. We assume that the film thickness, as determined by the size of the normally oriented molecule, remains constant (a reasonable approximation) and all that changes during monolayer formation is the molecular packing density. At small coverages, the molecules may have a small tilt which would alter the thickness marginally, but the influence of this on the calculation can be shown to be small. In the initial stages of growth, the adsorbed molecular density is very small and so is the effective refractive index, as expected from the LorentzLorenz formula (the effective refractive index being a weighted mean of the refractive index of air and the molecule in the SAM). As the monolayer grows to unity cove- rage, the packing density of the molecules increases as well as the refractive index. In the other model, the assumption that the film refractive index remains constant and that the film thickness changes from zero to molecular dimensions implies complete packing even at small times of growth of SAM (refractive index being taken to be the close-packed value) which is clearly unrealistic. In this approach, the QCM should sense complete mass coverage at very small times of growth since the thickness of the film on the quartz crystal plays no role in mass loading. Thus, the approach being currently employed by calculation of an effective ellipsometric thickness in addition to being unrealistic does not also correlate with QCM measurements. The above arguments in favour of the approach proposed is only to be expected since we have sought to use the Lorentz-Lorenz relationship, which models a physically realistic system of a mixture noninteracting substances.

Having said that the Lorentz-Lorenz formula applies only to non-interacting systems, what would be the problems associated with application of the formalism to SAMs? In SAM formation, the molecules chemisorb on the metal surface and this would effectively alter the dielectric properties of the metal surface and the use of a three-layer model for calculation of the film refractive index using the refractive index of the uncovered metal surface would not be strictly correct. This has been pointed out by Martensson and Arwin (1995) in their studies of protein adsorption on Au surfaces. Looking at this differently, while the interactions between the ODT molecules is expected to be predominantly Van der Waals, on chemisorption the molecules would interact with one another through the metal surface. Consequently, the thiolate group polarizability would change (as would the polarizability of the other groups in close proximity with the thiolate group through the induction effect) and the use of atomic molar refractivities in the molecule would also not be strictly correct. There is fair agreement between QCM and ellipsometry measurements of the system studied in this paper, but given the problems described above, that is not sufficient justification for the use of the approach. Further work is in progress in refining the above approach taking into account the above lacunae.

\section{Acknowledgements}

The author thanks Drs Vijaya Patil and K S Mayya, National Chemical Laboratory, Pune, for experimental assistance.

\section{References}

Azzam R M A and Bashara N M 1977 Ellipsometry and polarized light (New York: North Holland) 
Bain C D, Throughton E B, Tao Y T, Evall J, Whitesides G M and Nuzzo R G 1989 J. Am. Chem. Soc. 111321

Batsanov S S 1966 Refractometry and chemical structure (Princeton: Van Nostrand Applied Science Library) Ch. 1

Born M and Wolf E 1965 Principles of optics (New York: Pergamon Press)

Chen S H and Frank C W 1989 Langmuir 5978

Colvin V L, Goldstein A N and Alivisatos A P 1992 J. Am. Chem. Soc. 1145221

Cuypers P A, Corsel J W, Janssen M P, Kop J M M, Hermens W Th and Hemker H C 1983 J. Biol. Chem. 2582426

Den Engelsen D 1971 J. Opt. Soc. Am. 611460

Durand R R, Bencosme C S, Collman J P and Anson F C 1983 J. Am. Chem. Soc. 1052710

Frubose C and Doblhofer K 1995 J. Chem. Soc. Faraday Trans. 911949

Grabar K C et al 1996 Langmuir 122353

Hahner G, Woll C, Buck M and Grunze M 1993 Langmuir 91955

Honig E P and De Koning B R 1976 Surf. Sci. 56454
Kaeble D H 1971 Physical chemistry of adhesion (New York: Wiley-Interscience)

Kim M W, Sauer B B, Yu H, Yazdanian M and Zografi G 1990 Langmuir 6236

Lopez G P, Biebuyck H A, Harter R, Kumar A and Whitesides G M 1993 J. Am. Chem. Soc. 11510774

Martensson J and Arwin H 1995 Langmuir 11963

Notoya T and Poling Q W 1979 Corrosion 35193

Porter M D, Bright T B, Allara D L and Chidsey C E D 1987 J. Am. Chem. Soc. 1093559

Sauerbrey G 1959 Z. Phys. (Munich) 155206

Sun L, Johnson B, Wade T and Crooks R M 1990 J. Phys. Chem. 948869

Tillman N, Ulman A, Schildkraut J S and Penner T L $1988 \mathrm{~J}$. Am. Chem. Soc. 1116136

Vogel A, Cresswell W and Leicester J 1954 J. Phys. Chem. 58 174

Wasserman S R, Tao Y T and Whitesides G M 1989 Langmuir 51074 